\title{
Bilateral, Mirror-imaged, Postero-inferior Cerebellar Artery Aneurysms: Report of a Rare Case
}

Sharma GR, Jha R, Khadka N, Adhikari DR, Bista P, Sultaniya PK

Department of Neurosurgery, National Academy of Medical sciences, Bir Hospital ,Kathmandu, Nepal

\section{ABSTRACT}

A 60-year-old right-handed lady presented with the features of subarachnoid haemorrhage. The CT angiogram showed a pair of very rare bilateral, mirror-imaged distal postero-inferior cerebellar artery aneurysms. Both aneurysms were clipped via the midline posterior fossa craniectomy under general anaesthesia. The literatures is reviewed on the incidence, presentation, management and outcome of bilateral distal posterior-inferior cerebellar artery aneurysms.

Keywords: bilateral mirror image, distal postero-inferior cerebellar artery aneurysms, posterior fossa craniectomy, microsurgical clipping

\section{INTRODUCTION}

Of all the posterior circulation aneurysms, $20 \%$ originate from the postero-inferior cerebellar artery (PICA) and they account for about 0.5 - $3 \%$ of all intracranial aneurysms ${ }^{1,2}$. Any aneurysm arising from the peripheral part of the PICA other than the VA-PICA junction is a distal PICA aneurysm. The majority of PICA aneurysms arise from the vertebral artery-PICA junction. Only a much smaller percentage of PICA aneurysms arises from the distal segments of the PICA. The distal PICA aneurysms constitute only $0.28-1.4 \%$ of all intracranial aneurysms and they usually present with features of $\mathrm{SAH}$ (subarachnoid haemorrhage). Bilateralmirror imaged distal PICA aneurysms are very rare and, to our best of knowledge, only four cases have been reported in the literature. ${ }^{3}$ The diagnosis can be made by a cerebral angiogram.
The treatment of the distal PICA aneurysms is microsurgical clipping of the aneurysms, excision of the aneurysms or endovascular coiling. We hereby report a case of bilateral mirror-imaged distal PICA aneurysms and review the literature on distal PICA aneurysms.

\section{CASE REPORT}

A 60-year-old right-handed lady was admitted to our department with a history of a sudden onset of headache followed by altered sensorium. On examination, she was drowsy but obeying command and had no focal neurological deficit. Her laboratory parameters including coagulation profile were within normal limits. The CT scan of

\section{Correspondence:}

Dr. Gopal Raman Sharma

Department of Neurosurgery, National Academy

of Medical Sciences

Bir Hospital, Mahaboudha, Kathmandu, Nepal

GPO Box. NO. 19429

Phone. 4240058

Email: gopal47@hotmail.com 
the brain showed intraventricular blood, mainly in the $4^{\text {th }}$ ventricle, with an obstructive type of hydrocephalus (Figure 1).

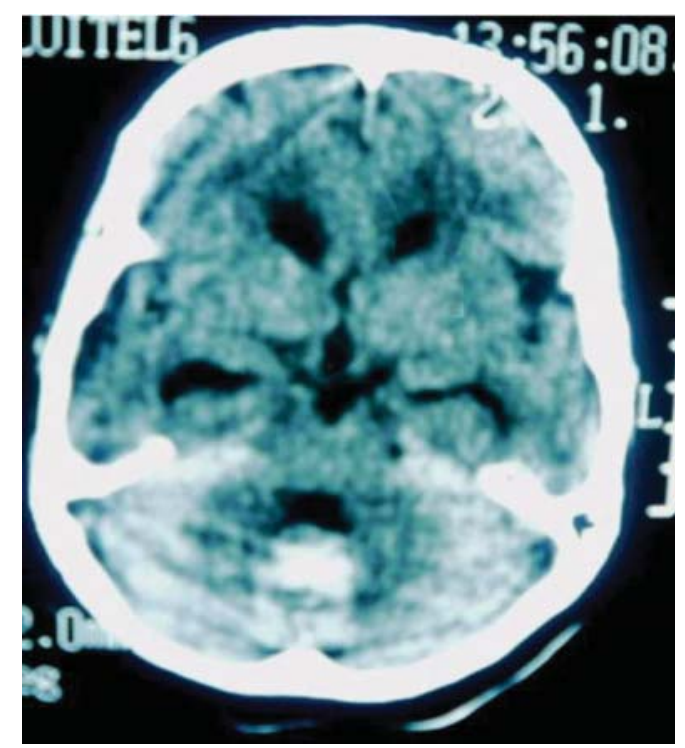

Figure 1. Preoperative CT scan of the brain showing blood in the fourth ventricle with obstructive hydrocephalus

The Distribution of blood on the CT scan was suggestive of a SAH due to a rupture of posterior circulation aneurysm. The lady was subjected to CT angiogram, which revealed bilateral distal PICA aneurysms. The right distal PICA aneurysm was $4.0 \times 6.2 \mathrm{~mm}$ in size with the neck of about $4.8 \mathrm{~mm}$ in width and the aneurysm was directed posteroinferiorly. The left distal PICA aneurysm was about $5.9 \times 4.0 \mathrm{~mm}$ in size with the neck about $3.5 \mathrm{~mm}$ in width and it was directed anterosuperiorly (Figure 2).

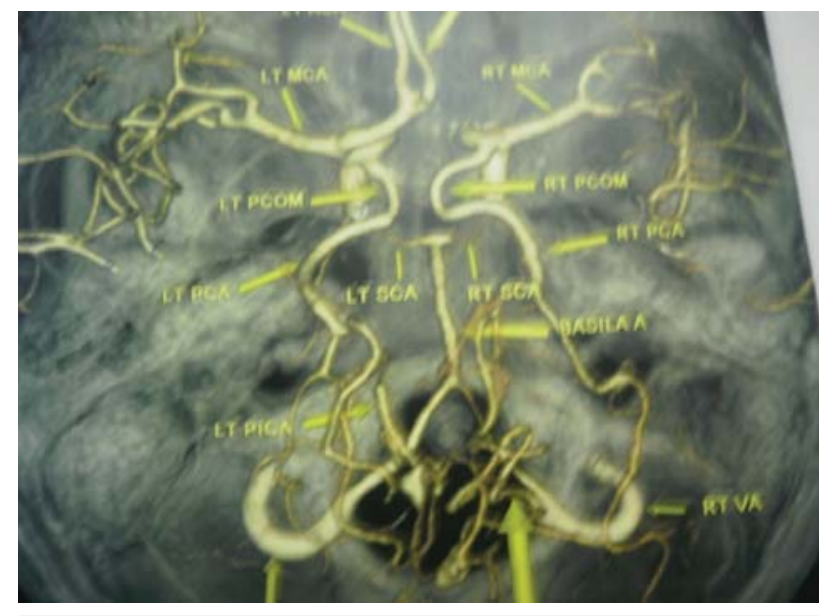

Figure 2. CT angiogram showing bilateral (mirrorimaged) distal PICA saccular aneurysms at the telovelotonsillar segments

On the $10^{\text {th }}$ day of ictus, she underwent a microsurgical clipping of both the distal PICA aneurysms via the midline suboccipital craniectomy. The right distal PICA aneurysm was irregular in shape, originated from the telovelotonsillar segment and was located in the medial aspect of the right cerebellar tonsil and there was no recent evidence of bleeding from this aneurysm. The left distal PICA aneurysm was irregular in shape, also originating from the left telovelotonsillar segment, situated in the roof of the $4^{\text {th }}$ ventricle and the aneurysm was surrounded by clots and appeared that the bleeding was by a rupture of this aneurysm. The necks of the aneurysms were occluded using $6 \mathrm{~mm}$ straight titanium Sugita clips.

On the first postoperative day, the patient was obeying commands and had no neurological deficit. On the third postoperative day, she gradually became unconscious. The CT angiogram revealed hydrocephalus and complete occlusion of both the aneurysms with obliteration of the sacs and the clips in situ (Figure 3 and 4).

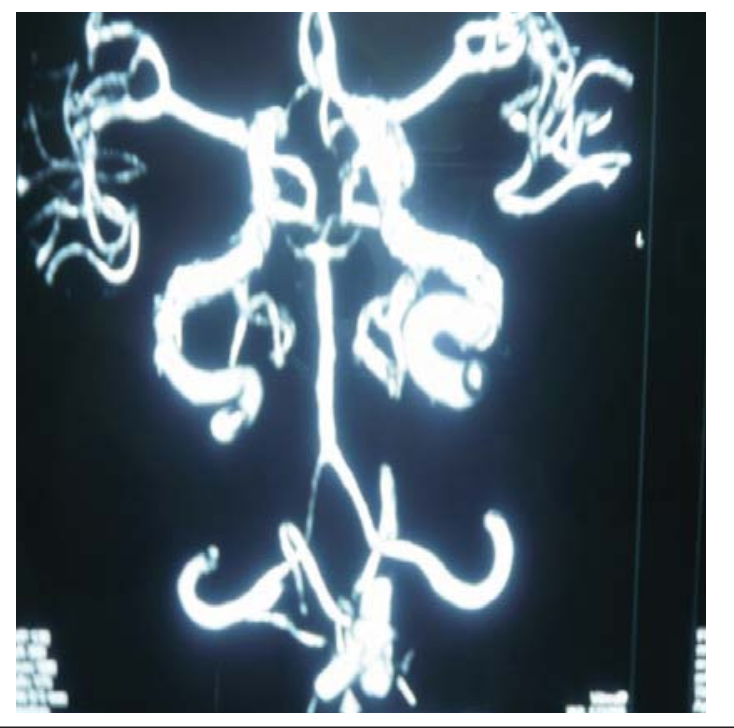

Figure 3. CT angiogram depicting total occlusion of the neck of both the distal PICA aneurysms and obliteration of the sacs. The clips are in situ.

An emergent ventriculo-peritoneal shunt (VP) was performed. The cerebro spinal fluid collected peroperatively was within normal limits. After the VP shunt, she regained consciousness. On the sixth postoperative day, she again deteriorated neurologically and the scan was repeated, which showed posterior fossa extradural haematoma (EDH) amounting to $15 \mathrm{ml}$. The EDH was evacuated and she gradually improved and started obeying commands. On the ninth postoperative day, she again became unconscious and was subjected to another CT scan of the brain which revealed fresh intraventricular bleeding. All necessary laboratory tests were carried out to rule out coagulopathy. The results of the coagulation profiles were suggestive of dissiminated intravascular coagulation. On the $22^{\text {nd }}$ 
postoperative day, the coagulation profile came to the normal level. The CT scan of the $27^{\text {th }}$ postoperative day showed features of VP shunt obstruction and a shunt-was exteriorized. The report of the CSF analysis was normal and a shunt-revision was performed on the $28^{\text {th }}$ postoperative day. On the $32^{\text {nd }}$ postoperative day, she developed a high-grade fever. The central line tip culture showed a growth of Staphylococcus aureus and Acinetobactor was isolated in the sputum culture. Antibiotics were administered according to the culture and sensitivity. On the $35^{\text {th }}$ postoperative day, she deteriorated and developed features of acute respiratory distress syndrome and was put on a mechanical ventilator. On the $40^{\text {th }}$ postoperative day, she succumbed to acute respiratory distress syndrome with septicemia.

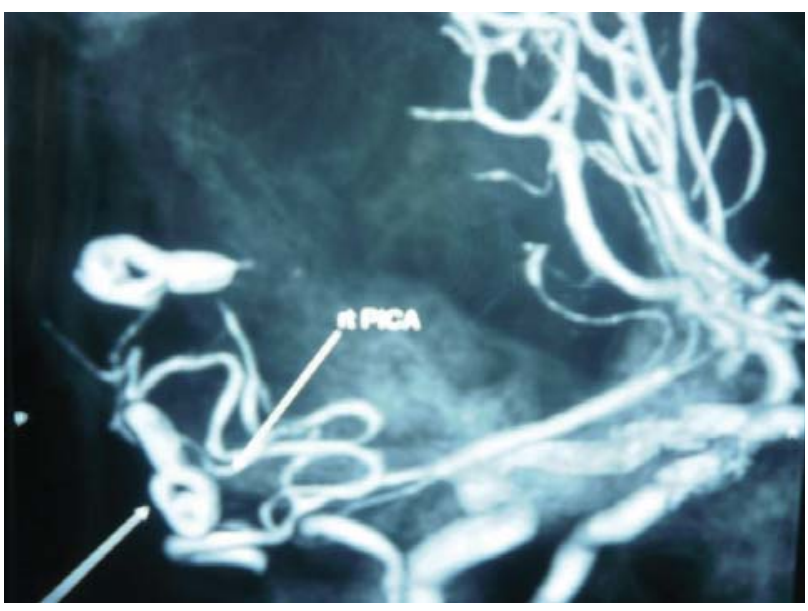

Figure 4. The CT angiogram (lateral view) revealed complete obliteration of the sacs of both the distal PICA aneurysms. The clips are in the normal positions.

\section{DISCUSSION}

The posterior inferior cerebellar artery is divided into five segments: anterior medullary, lateral medullary, tonsillomedullary, telovelotonsillar and cortical. These divisions are important for the surgical plan for distal PICA aneurysms ${ }^{4}$. The first three segments of the PICA give perforating branches to the brain stem, so that a sacrifice of the PICA within these segments can lead to a brain stem infarct. The rare distal PICA aneurysm that may arise in the telovelotonsillar and cortical segments can be excised together with the parent vessel if necessary ${ }^{5}$. The age and sex distribution of patients with distal PICA aneurysms are similar to those of other intracranial aneurysms in that they are more common in females than males and occur more in the middle and in old age $^{4,6}$.

The symtoms of distal PICA aneurysms are similar to those of SAH, like sudden onset of headache, vomiting followed by decreased level of consciousness and nuchal rigidity $2,7,8,9,10$. The literature reviewed reveals that all the reported cases of bilateral distal PICA aneurysms had features of SAH on admission ${ }^{3}$. The first choice of investigation to diagnose SAH due to ruptured distal PICA aneurysms is a CT scan of the brain that shows that the blood is mainly distributed in and around the fourth ventricle. The majority of patients will have an obstructive type of hydrocephallus due to the intraventricular blood $3,5,8,11$. CT findings of $\mathrm{SAH}$ due to bilateral distal PICA aneurysms are similar to those of the distal PICA aneurysm bleed. The final diagnosis of bilateral distal PICA aneurysms is made by a cerebral angiogram. The four vessels cerebral angiogram is the gold standard diagnostic tool. The CT angiogram is another choice of investigation ${ }^{6,8}$.

Distal PICA aneurysms may be small to quite large. However, no giant aneurysm was found in the reported cases of bilateral distal PICA aneurysms ${ }^{3}$. Although the most common type in distal PICA is saccular, fusiform and dissecting aneurysms are also described in the literature ${ }^{5}$. Only saccular aneurysms were found in the reported cases of bilateral distal PICA aneurysms.

Similar to other distal PICA aneurysms, treatment of bilateral distal PICA aneurysms is either surgery or endovascular procedure $5,6,8,12,13$. The surgical treatment of distal PICA aneurysms includes microsurgical clipping, trapping, excision and ligation ${ }^{3,7}$. Aneurysms which are located in the anterior and lateral medullary segments are approached via the lateral suboccipital and tosillomedullary, telovelotonsillar and cortical segments are approached via the midline suboccipital. Microsurgical clipping is the best surgical option, however, sometimes, aneurysms located in the telovelotosillar and cortical segments can be trapped, excised and ligated since no medullary branches come out from these segments. In our case, both the aneurysms were in the telovelotonsillar segments and were clipped via midline suboccipital approach.

The distal PICA aneurysms are more superficial than other posterior circulation aneurysms, and their surgical outcomes have been reported to be excellent because of the small amount of intraparenchymal injury in the adjacent areas and the relatively straightforward surgical approach. Though the surgical outcome was good, our patient succumbed to other complications. Lewis SB et al operated on 20 patients for 22 distal PICA aneurysms, with $85 \%$ good results ${ }^{8}$. The operative results of bilateral distal PICA aneurysms are similar to that of other distal PICA aneurysms. There was no mortality in all the four reported cases and the surgical outcome was excellent ${ }^{3}$.In case where the surgical treatment is relatively difficult or in which the risks for 
general anaesthesia or surgery are great, endovascular coiling is indicated. Dowd et al treated three cases of aneurysms of distal PICA with GD coiling and reported excellent results ${ }^{12}$.

\section{REFERENCES}

1. Lister JR, Rhoton AL jr, Matsushima T, et al. Microsurgical anatomy of the posterior inferior cerebellar artery. Neurosurgery.1982;10: 170.

2. Peluso JP, Van Rooij WJ, Sluzewski M, Beute GN, Majoie CB. Posterior inferior cerebellar artery aneurysms : Incidence, clinical presentation, and outcome of endovascular treatment. Am J Neuroradiol. 2008;29:86-90.

3. Sano K, Hayano M, Ibuchi Y . A case of distal aneurysms of bilateral posterior inferior cerebellar arteries. No Shinkei Geka. 1993;21(7):645.

4. Tokimura H, Yamahato h, Kamezawa T, Tajitsu K, Nagayama $\mathrm{T}$, Sugata s, et al. Clinical presentation and treatment of distal posterior inferior cerebellar artery aneurysms. Neurosurg Rev. 2011;34(1):57-67.

5. Park JS, Lee TH, Seo EK, Cho YJ. Aneurysms of distal posterior inferior cerebellar artery. J Korean Neurosurg Soc. 2008;44(4). 205-10.

6. Anegawa S, Hayashi T, Torigoe R et al .Aneurysms of the distal posterior inferior cerebellar artery - analysis of 14 aneurysms in 13 cases. J No Shinkei Geka. 2001;29:121-9.
7. Lewis SB , Chang DJ, Peace DA, Lafrentz PJ, Day AL. Distal posterior inferior cerebellar artery aneurysms: clinical features and management. J Neurosurg. 2002; 97.

8. Li XE, Wang YY, Li G, Jia DZ, Liu XH, Goa J , Li XG. Clinical presentation and treatment of distal posterior inferior cerebellar artery aneurysms: Report on five cases. Surg Neurol. 2008;70(4):425-30.

9. Mishino A, Sakurai $Y$, Satoh, $\mathrm{H}$ et al. Aneurysms of the distal posterior inferior cerebrllar artery: report of 10 cases. No Shinkei Geka. 1991;19:925-32.

10. Demirgil B , Gunaldi O, Tugcu B, Postalci L, Colluoglu B, Tanriverdi $\mathrm{O}$, et al . Multiple aneurysms of distal posterior inferior cerebellar artery: two case reports.Minim Invasive Neurosurg. 2008;51(5):249-52.

11. Dowd CF, Halbach VV, Higashida RT, Barwell SL, Hieshima GB. Endovascular coil embolization of unusual posterior inferior cerebellar artery aneurysms.Neurosurgery. 1990;27:954-61.

12. Mariniello G, Briganti F, Fabio T, Merseglia M, Mairu F. Bilateral Mirror aneurysms at the origin of the posterior inferior cerebellar artery: case report and review of literature. J Neurolintrevent Surg. 2010; 2:157-9. 